\title{
CCEREM: Critical-Convergence Extended-Resolution Electron Microscopy
}

\author{
Michael A. O'Keefe, * Lawrence F. Allard,** Douglas A. Blom*** \\ *OKCS, 18528 Mesa Verde Way, Castro Valley, CA 94552 \\ ** Materials Science \& Technology Div., Oak Ridge National Lab, Oak Ridge, TN 37831 \\ ***EM Center, University of South Carolina, 715 Sumter St, Columbia, SC 29208
}

A high-resolution transmission electron microscope (HRTEM) passes information on atom positions from specimen to microscope image as specimen-generated spatial frequencies constrained by two limits: the microscope resolution limit and its information limit [1]. Microscope information limit (MIL) is the high-frequency limit on the specimen's structural information that the microscope can deliver to the image. Microscope resolution limit (MRL) is the high-frequency limit on information delivered with same-sign phase [2]. Critical-convergence extended-resolution electron microscopy (CCEREM) is a method of extending HRTEM resolution from a microscope's spherical-aberrationlimited Scherzer resolution [2] to its temporal-coherence-limited information limit.

Specimen information at frequencies higher than the native Scherzer resolution imposed by inherent spherical aberration $\left(\mathrm{C}_{\mathrm{S}}\right)$ of the objective lens has been obtained by adding hardware correctors with negative spherical aberration [3], by hardware/software methods such as holography [4], or from focal series of images by purely software methods, including comparison of experimental images with ones simulated from model structures [5] or reconstruction of the specimen exit-surface wave from focal series [6,7]. Linear transfer from the electron wave at the specimen exit surface to the image is shown by the phase contrast transfer function (CTF). Figure 1 shows CTFs for a typical non- $\mathrm{C}_{\mathrm{S}}$-corrected HRTEM. Figure 2 shows these CTFs when incident beam convergence is set at the critical value. By taking a focal series of images under CCEREM conditions, it is possible to construct an extended virtual critical-convergence CTF merely by adding images obtained at values of defocus corresponding to higher passbands - adding images in real space is equivalent to adding their CTFs in Fourier space. Critical convergence is chosen both to minimize unwanted transfers and to overlap desired transfers to produce a continuous CCEREM CTF (Fig. 3).

As well as the extended resolution offered by the composite CCCTF, an equally important property of CCEREM is its elimination of delocalization. Delocalization and convergence damping are both strongest where the slope of the CTF is steepest [8], so CCEREM acts as a "delocalization filter", passing spatial frequencies only where their delocalization is minimal. Critical convergence also strongly damps second-order image contributions [9], delivering a much truer structure image. Experimentally, CCEREM requires that electrons be incident on the specimen in a cone with a semiangle much larger than normally used for HRTEM. Critical convergence may be implemented by filling a large condenser aperture (possibly requiring an additional condenser lens) or by spinning an incident beam in a spiral pattern to create a cone. Defocus stepping and image collection could be automated. The composite image is formed by simple addition, which could be performed at the image detector, requiring only one image collection per focal series. With sufficiently rapid changes in defocus (by small steps in lens current or accelerating voltage or piezoelectric specimen height) it may even be possible to generate composite CCEREM images at real-time (video) rates [10].

[1] M.A. O'Keefe, L.F. Allard and D.A. Blom, Microscopy \& Microanalysis 16 S02 (2010) 766-767.

[2] O. Scherzer, J. Applied Physics 20 (1949) 20-28.

[3] M. Haider, G. Braunshausen \& E. Schwan, Optik, 99 (1995) 167-179.

[4] H. Lichte, Ultramicroscopy 38 (1991) 13-22.

[5] M.A. O'Keefe, P.R. Buseck and S. Iijima, Nature 274 (1978) 322-324.

[6] W.M.J. Coene, A. Thust, M. Op de Beeck and D. Van Dyck, Ultramicroscopy 64 (1996) 109-135.

[7] A. Thust, W.M.J. Coene, M. Op de Beeck and D. Van Dyck, Ultramicroscopy 64 (1996) 211-230.

[8] M.A. O'Keefe, Ultramicroscopy 1083 (2008) 196-209. doi:10.1016/j.ultramic.2007.07.009.

[9] M.A. O'Keefe 37th Ann. Proc. EMSA, San Antonio, Texas (1979) 556-557.

[10] Research at Oak Ridge National Laboratory's High Temperature Materials Laboratory sponsored by U.S. Department of Energy, Office of Energy Efficiency and Renewable Energy, Vehicle Technologies program. 


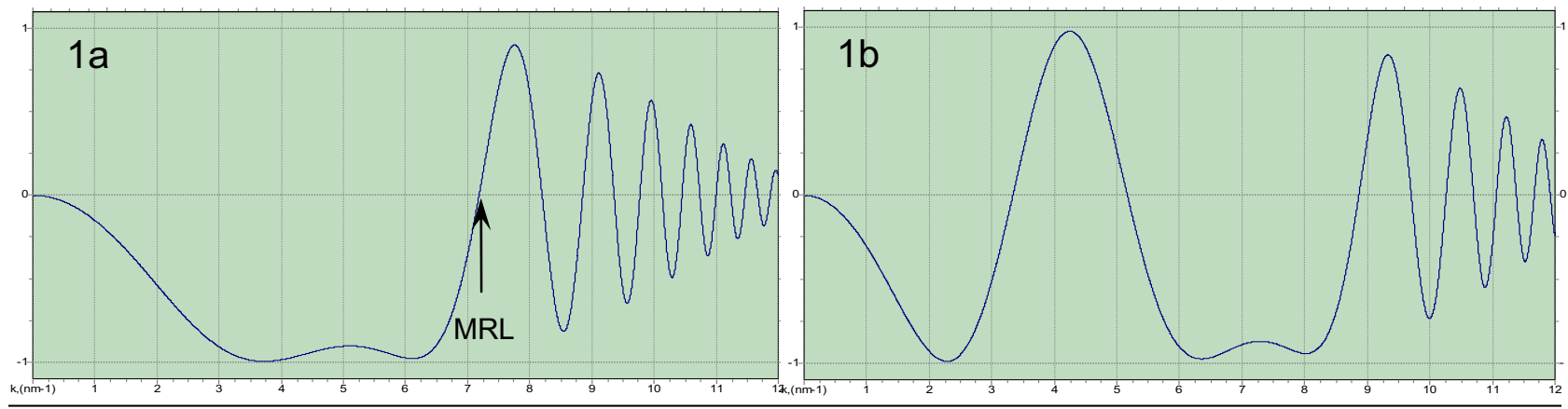

FIG.1. Phase contrast transfer functions display linear transfer of spatial frequencies from specimen exit-surface wave to image. Resolution limit (MRL) is at the first Scherzer defocus zero crossing (a). Second passband defocus (b) has low-frequency zero crossings. Oscillations in CTFs are indicative of large delocalizations when corresponding spatial frequencies contribute to the image.
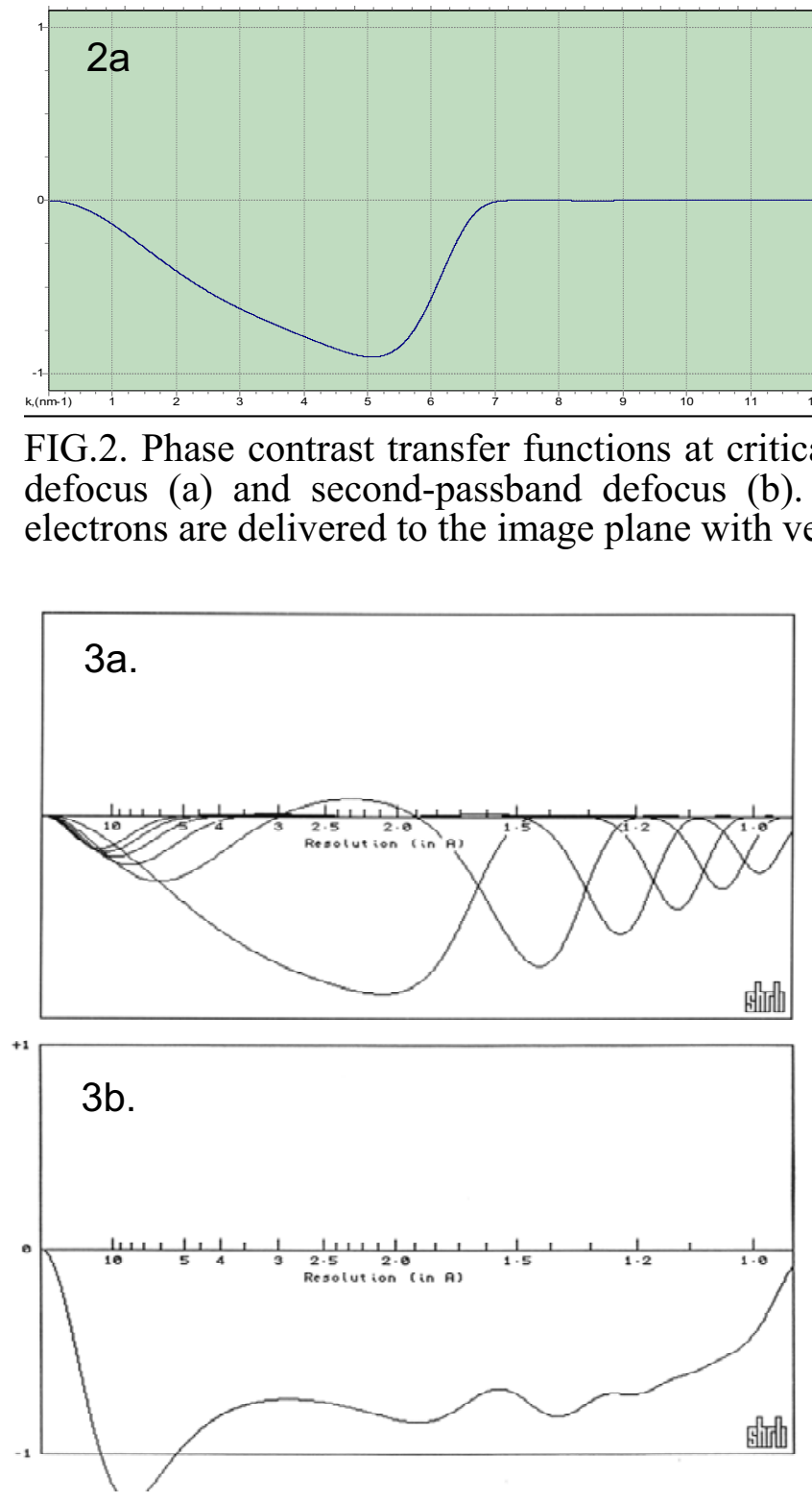

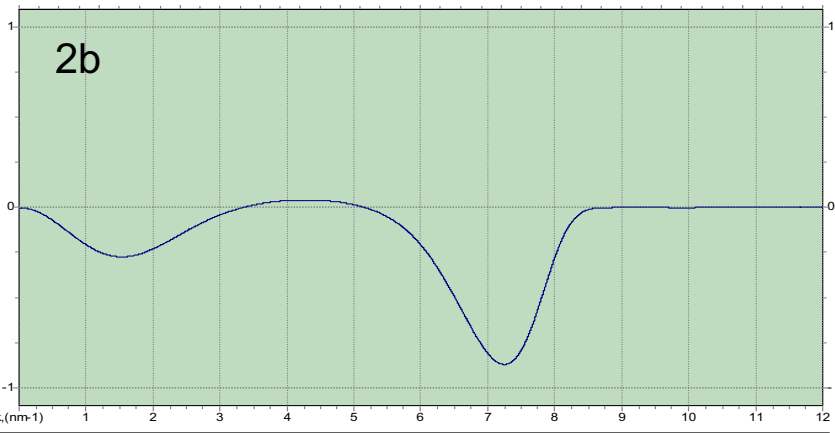

convergence show no zero crossings at Scherzer Elimination of oscillations means that imaging ery low delocalization.

FIG. 3. (a) First six passbands under CCEREM conditions show transfer of successively higher spatial frequencies with minimal delocalization.

(b) Simple summation of the first six passbands gives an extended virtual CTF that shows how spatial frequencies making up the composite CCEREM image are transferred from the specimen exit-surface wave to the image. Because it is assembled from a series of low-delocalization CCEREM passbands, the composite CCEREM CTF (CCCTF) ensures minimal delocalization in the corresponding composite CCEREM image.

The CCCTF readily passes information into the composite image at the low frequency end, in contrast to CTFs from $\mathrm{C}_{\mathrm{S}}$-corrected HRTEMs with extremely-low values of effective spherical aberration. CCEREM could be used to add lowfrequency non-delocalized information to images from $\mathrm{C}_{\mathrm{S}}$-corrected microscopes by adjusting $\mathrm{C}_{\mathrm{S}}$ to some fraction of the native $\mathrm{C}_{\mathrm{S}}$.

\section{<sub-Angstrom@comcast.net>}

